\title{
Long-range charge order in the low-temperature insulating phase of $\mathrm{PrNiO}_{3}$
}

\author{
M. Medarde, ${ }^{1}$ M. T. Fernández-Díaz, ${ }^{2}$ and Ph. Lacorre ${ }^{3}$ \\ ${ }^{1}$ Laboratory for Developments and Methods, Paul Scherrer Institute, CH-5232 Villigen PSI, Switzerland \\ ${ }^{2}$ Institut Laue-Langevin, BP 156, 38042 Grenoble Cedex 9, France \\ ${ }^{3}$ Laboratoire des Oxydes et Fluorures, Faculté des Sciences et Techniques, Université du Maine, Av. Olivier Messiaen, \\ F-72085 Le Mans Cedex 9, France
}

(Received 9 October 2008; published 16 December 2008)

\begin{abstract}
We report the observation of a static long-range $2 \mathrm{Ni}^{3+} \rightarrow \mathrm{Ni}^{3+\delta}+\mathrm{Ni}^{3-\delta}$ charge redistribution in $\mathrm{PrNiO}_{3}$ below $T_{\mathrm{MI}}=130 \mathrm{~K}$ by means of high-resolution powder neutron diffraction. In contrast to previous structural studies, these present data show that the symmetry of the low-temperature insulating phase is not orthorhombic but monoclinic $P 2_{1} / n$. The two available $\mathrm{Ni}$ sites host contracted and expanded $\mathrm{NiO}_{6}$ octahedra which alternate along the three pseudocubic perovskite axes, suggesting the existence of a $\mathrm{Ni}^{3+\delta} / \mathrm{Ni}^{3-\delta}$ charge order similar to that reported for the nickelates with heavier lanthanide ions. The difference between the average Ni-O distances in the two Ni sites at $T / T_{\mathrm{MI}}=0.08$ is $0.052(2) \AA$, which is almost two times smaller than the value reported for $\mathrm{LuNiO}_{3}$ at $T / T_{\mathrm{MI}}=0.50[0.084(4) \AA]$. This result suggests a progressive decrease in $\delta$ by approaching the itinerant limit and gives further support to the charge disproportionation mechanism as an origin of the metal-insulator transition in the whole $\mathrm{RNiO}_{3}$ family.
\end{abstract}

DOI: 10.1103/PhysRevB.78.212101

PACS number(s): 71.27.+a, 61.05.fm, 71.30.+h, 71.45.Lr

The mechanism of the gap opening in the Ni perovskite family $R \mathrm{NiO}_{3}(R=\mathrm{Y}$ and rare earth $\neq \mathrm{La})$ has been the subject of an increasing number of investigations since its discovery in $1991 .{ }^{1}$ With the exception of metallic $\mathrm{LaNiO}_{3}$, all the members of the series undergo a first-order metal-toinsulator (MI) transition at temperatures $T_{\mathrm{MI}}$ ranging between $130(\mathrm{Pr})$ and $600 \mathrm{~K}(\mathrm{Lu}){ }^{1,2}$ The electronic localization is accompanied by subtle structural changes involving the $\mathrm{Ni}-\mathrm{O}$ distances and the Ni-O-Ni angles whose fine details, not yet fully elucidated, are still a matter of controversy. ${ }^{2-6}$

In contrast to earlier structural studies, the recent availability of the heavier nickelates made possible the observation of a symmetry decrease from orthorhombic Pbnm to monoclinic $P 2_{1} / n$ below $T_{\mathrm{MI}}$ for some of these compounds $(R=\mathrm{Ho}, \mathrm{Y}, \mathrm{Er}, \mathrm{Tm}$, and $\mathrm{Lu}) .^{2}$ The splitting of the unique nominally $\mathrm{Ni}^{3+}$ site present in the metallic state into two inequivalent $\mathrm{Ni1}$ and $\mathrm{Ni} 2$ sites with slightly different average $\mathrm{Ni}-\mathrm{O}$ distances was interpreted as the signature of a charge disproportionation (CD) of the type $2 \mathrm{Ni}^{3+} \rightarrow \mathrm{Ni}^{3+\delta}+\mathrm{Ni}^{3-\delta}$. This mechanism, which contradicted previous chargetransfer and negative-delta gap models, ${ }^{3,7}$ was fairly unexpected for a $3 d^{7}$ system. Although CD is frequently encountered in compounds containing broad-band $6 s^{1}$ ions such as $\mathrm{Bi}^{4+}, \mathrm{Pb}^{3+}$, or $\mathrm{Tl}^{2+}$ due to their strong tendency to form $6 s^{0}$ $+6 s^{2}$ closed shells, it is rather uncommon and a priori much more expensive in narrow-band $3 d$ transition-metal (TM) oxides where Mott-Hubbard repulsion usually prevents charge transfer between metallic sites. Interestingly, the few $3 d$ oxides for which the existence of $\mathrm{CD}$ has been reported are exclusively $e_{g}^{1}$ orbitally degenerate systems close to the boundary between localized and itinerant behaviors [such as $\mathrm{CaFeO}_{3}\left(t_{2 g}^{3} e_{g}^{1}\right),{ }^{8} \mathrm{AgNiO}_{2}\left(t_{2 g}^{6} e_{g}^{1}\right),{ }^{9}$ or the $R \mathrm{NiO}_{3}$ family]. The occurrence of the $e_{g}^{1} e_{g}^{1} \rightarrow e_{g}^{0} e_{g}^{2}$ process in these compounds has been recently interpreted as an alternative to conventional Jahn-Teller (JT) distortions. ${ }^{7,10}$

Within this framework, larger bandwidths are expected to reduce the $\mathrm{JT}$ effect, and at the same time, to ease the charge transfer between TM sites. In the particular case of the $R \mathrm{NiO}_{3}$ family, less distorted $\mathrm{NiO}_{6}$ octahedra and a more complete CD would be expected by approaching the itinerant limit. Experimentally, only the first trend has been observed. The strength of the $\mathrm{CD}$, as measured by $\delta$, is larger for the most insulating nickelates ( $\mathrm{Lu}$ to $\mathrm{Ho}, \sim 0.35 e^{-}$) whereas for the more conducting ones (Dy to Pr), attempts to observe the associated long-range charge order by x-ray or neutron diffraction have been unsuccessful. In spite of compelling evidence for a symmetry decrease from other techniques, ${ }^{11-14}$ no diffraction studies, even on single crystals, ${ }^{15,16}$ could provide full structure refinements supporting the existence of $\mathrm{CD}$ for the early nickelates. Although a larger $\delta$ could be compatible with less pronounced structural changes if the transferred electrons are progressively delocalized out of the $\mathrm{Ni}-\mathrm{O}$ bonds for increasing $\mathrm{Ni} e_{g}-\mathrm{O} 2 p$ hybridization, recent extended $\mathrm{x}$-ray-absorption fine structure (EXAFS) and $\mathrm{x}$-ray absorption near-edge structure (XANES) measurements ${ }^{5,6}$ suggest instead the existence of large but local (short-range) distortions. The giant ${ }^{16} \mathrm{O}-{ }^{18} \mathrm{O}$ isotope effect on $T_{\mathrm{MI}}$ and the strong decrease in the lattice contribution to the thermal conductivity below this temperature suggest also that the distortions could have an important dynamic component. ${ }^{17,18}$

The motivation of the present work was to determine the precise boundary of a static long-range $\mathrm{CD}$ and to explore its coexistence with local and/or dynamic structural distortions. For this purpose, we decided to reinvestigate the crystallographic structures of the early nickelates. Here we report high-resolution data on $\mathrm{PrNiO}_{3}$, the first member of the series, measured on the diffractometer D2B [Ge (335), $\lambda$ $=1.594 \AA]$ at the Institute Laue Langevin in Grenoble (France). A large (6 g) sample was prepared as described in Ref. 1 . In contrast to most high-pressure synthesized materials, it displayed an excellent crystallinity and was nearly free of impurities $(1 \% \mathrm{NiO})$. Thermogravimetric measurements indicated an oxygen content of $3+\delta$ with $\delta=0.00(0)$, very close to the results obtained from neutron diffraction $[\delta$ 
TABLE I. Refined structural parameters of $\mathrm{PrNiO}_{3}$ at $T=10 \mathrm{~K}$ using the space group $P 2_{1} / n$ with cell parameters $a=5.41139(2) \AA, b=5.38216(3) \AA$, $c=7.61141(4) \AA$, and $\beta=90.0715(5)$.

\begin{tabular}{lllllcc}
\hline \hline Atom & W. Pos. & Occ & $x$ & $y$ & $z$ & $\begin{array}{c}\text { Biso } \\
\left(\AA^{2}\right)\end{array}$ \\
\hline $\mathrm{Ni} 1$ & $2 d$ & 0.5 & $1 / 2$ & 0 & 0 & $0.16(1)$ \\
$\mathrm{Ni} 2$ & $2 c$ & 0.5 & $1 / 2$ & 0 & $1 / 2$ & $0.16(1)$ \\
$\mathrm{Pr}$ & $4 e$ & 1.0 & $0.9946(5)$ & $0.0327(4)$ & $0.2510(8)$ & $0.19(3)$ \\
$\mathrm{O} 1$ & $4 e$ & $1.030(1)$ & $0.0704(3)$ & $0.4926(4)$ & $0.2528(5)$ & $0.20(2)$ \\
$\mathrm{O} 2$ & $4 e$ & $0.982(3)$ & $0.7145(6)$ & $0.2784(9)$ & $0.0365(5)$ & $0.37(6)$ \\
$\mathrm{O} 3$ & $4 e$ & $1.055(3)$ & $0.2219(7)$ & $0.214(1)$ & $0.9635(5)$ & $0.40(6)$ \\
\hline \hline
\end{tabular}

$=0.067(7)$, see Table I]. Several neutron powder-diffraction patterns were recorded by warming between 10 and $170 \mathrm{~K}$ and analyzed using the Rietveld program FULLPROF. ${ }^{19}$ A representative pattern is shown in Fig. 1(a).

Figures 1(b)-1(e) show two high-angle regions of the patterns measured at 170 and $10 \mathrm{~K}$. Although the hightemperature data could be satisfactorily fitted using the space group Pbnm proposed in previous works [Figs. 1(b) and 1(c)], the extra splitting of some of the reflections in the low-temperature pattern could not be reproduced. This split-

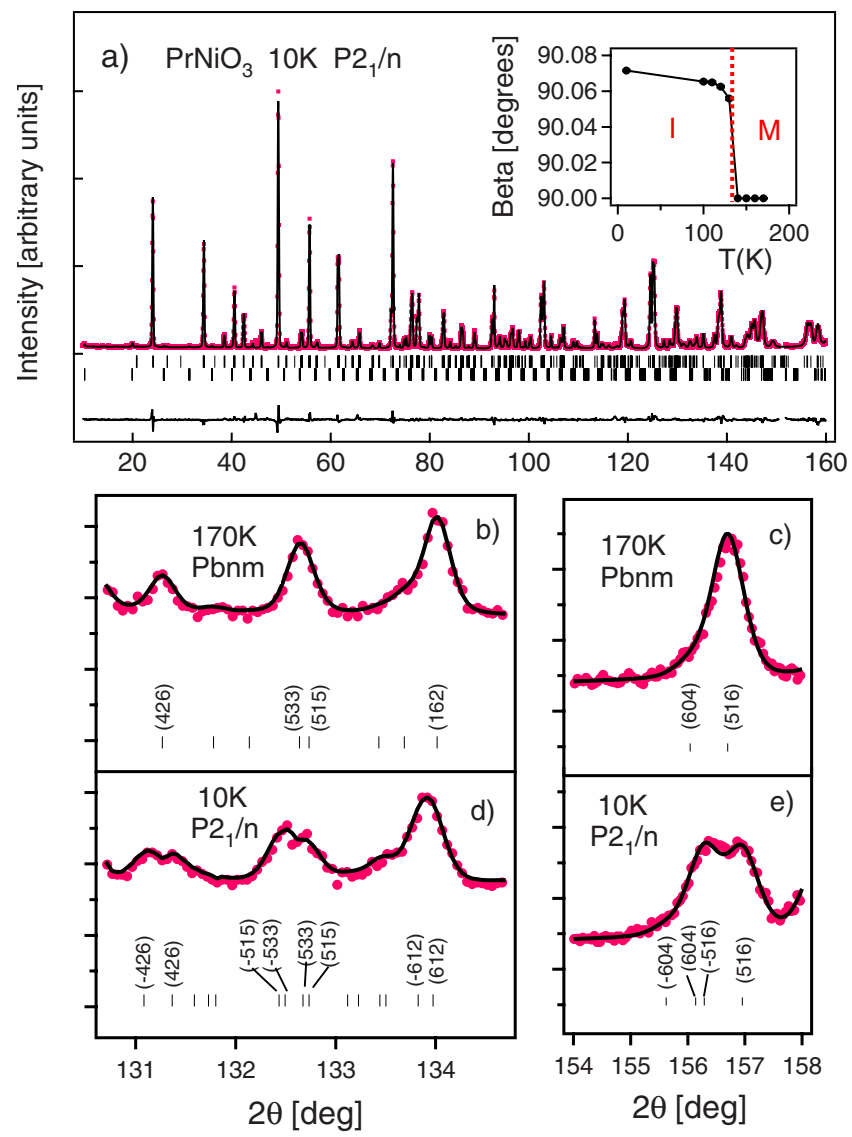

FIG. 1. (Color online) (a) Best fit of the neutron powderdiffraction pattern of $\mathrm{PrNiO}_{3}$ at $10 \mathrm{~K}$ using the space group $P 2_{1} / n$. Inset: temperature dependence of the monoclinic angle $\beta$. (b) and (c) Detail of the high- $2 \theta$ region of the pattern measured at $170 \mathrm{~K}$ and fitted with the space group Pbnm. (d) and (e) Same region measured at $10 \mathrm{~K}$ and fitted using the space group $P 2_{1} / n$. ting, not observed in previous works presumably because of lack of resolution and/or sample-related effects (size or strain broadening), strongly suggested the existence of a static long-range symmetry change below $T_{\mathrm{MI}}$. Figures $1(\mathrm{~d})$ and 1 (e) show the fit of the $10 \mathrm{~K}$ pattern refined using the space group $P 2_{1} / n$ proposed for the low-temperature phase of the nickelates with smaller rare earths. The splitting can be well described now even at the largest measured $2 \theta$ angle $u$ sing the same resolution function as in the high-temperature metallic phase. This last point indicates that short-range charge ordering, if existing, coexists with the long-range CD, concerns only a very small sample volume and has a coherence length below the detection limit of neutron powder diffraction $(40-50 \AA)$. The refinement of the full $10 \mathrm{~K}$ pattern and the temperature dependence of the monoclinic angle $\beta$ are shown in Fig. 1(a), and a summary of the refined cell parameters, atomic coordinates, and isotropic temperature factors at $10 \mathrm{~K}$ is displayed in Table $\mathrm{I}$.

The temperature dependence of the refined Ni-O distances is displayed in Fig. 2(a). A clear splitting in two wellseparated sets is observed below $T_{\mathrm{MI}}$. The distortion of the $\mathrm{NiO}_{6}$ octahedra, nearly absent in the metallic state, increases

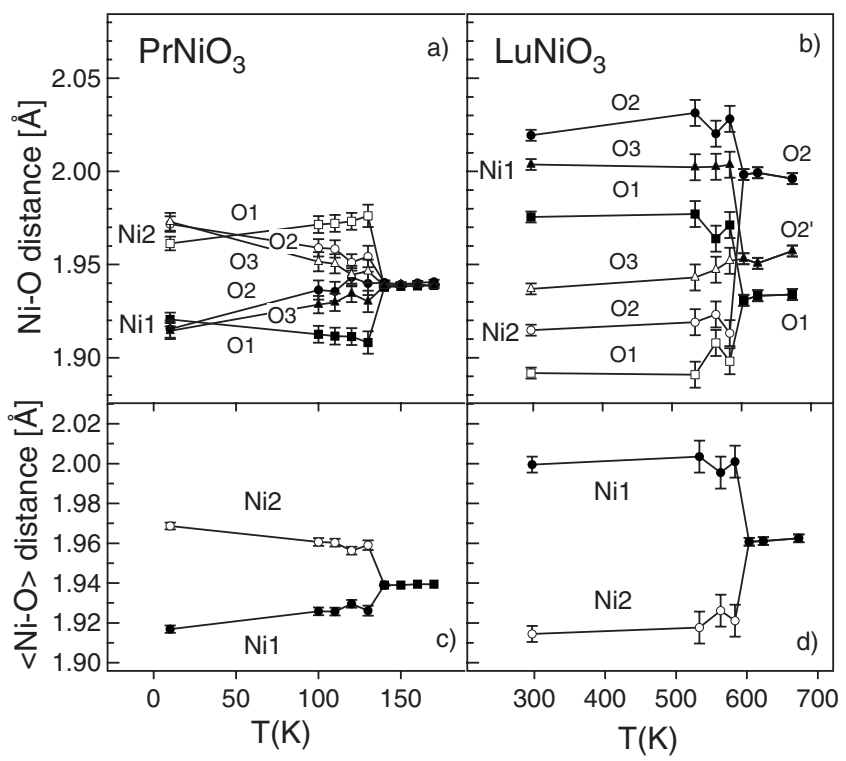

FIG. 2. (a) and (c) Temperature dependence of the individual and site-averaged Ni-O distances across $T_{\mathrm{MI}}$ for $\mathrm{PrNiO}_{3}$. (b) and (d) The same data for $\mathrm{LuNiO}_{3}$ (adapted from Ref. 2). 
slightly just below $T_{\mathrm{MI}}$ but decreases again by lowering the temperature for both the $\mathrm{Ni} 1$ and $\mathrm{Ni} 2$ sites. This behavior contrasts with that reported for the end member of the series $\mathrm{LuNiO}_{3}$, where the octahedra are strongly distorted in both the metallic and the insulating phases [see Fig. 2(b)]. ${ }^{2}$ The value of the distortion parameter $\Delta_{d}=(1 / 6) \sum_{n=1,6}\left[\left(d_{n}\right.\right.$ $-\langle d\rangle) /\langle d\rangle]^{2}$ is even larger above $T_{\mathrm{MI}}$, a trend also reported for the nickelates with $R=\mathrm{Ho}, \mathrm{Y}$, and $\mathrm{Er}^{2}$ Since JT distortions are expected to be absent in metals, the very similar bond-length dispersions at both sides of the transition suggest the existence of a stronger rare-earth-dependent steric component superimposed to a possible-in any case much smaller-JT distortion.

The evolution of the average Ni-O distances across the transition is shown in Fig. 2(c) and 2(d). For $\mathrm{PrNiO}_{3}$ the single Ni site in the metallic state $(1.9390 \AA$ at $150 \mathrm{~K})$ splits into Ni1 (contracted) and $\mathrm{Ni} 2$ (expanded) with average Ni-O distances of 1.9168 and $1.9687 \AA$ at $10 \mathrm{~K}$. The difference $\Delta d=0.052 \AA$ is slightly smaller than that measured by EXAFS for $\mathrm{NdNiO}_{3}(\sim 0.09 \AA)$ but is not inconsistent due to the lower accuracy of the last technique. ${ }^{5}$ In the case of $\mathrm{LuNiO}_{3}$, both the value of the average Ni-O distance in the metallic state $(1.9624 \AA$ at $673 \mathrm{~K})$ and the splitting of the two Ni sites in the insulating state $\left(d_{\mathrm{Ni1}-\mathrm{O}}=1.999 \AA, d_{\mathrm{Ni2}-\mathrm{O}}\right.$ $=1.915 \AA$, and $\Delta d=0.084 \AA$ at $\mathrm{RT}$ ) are significantly larger than for Pr even at higher values of $T / T_{\mathrm{MI}}$ [see Fig. 2(d)]. Another important difference between the two end members of the series is the exchange of the expanded and contracted sites (see Fig. 2 and Table I). This is presumably due to the crossing of the $\mathbf{a}$ and $\mathbf{b}$ cell parameters for $R=\mathrm{Nd}(\mathbf{a}>\mathbf{b}$ for $\operatorname{Pr}, \mathbf{a} \sim \mathbf{b}$ for $\mathrm{Nd}$, and $\mathbf{a}<\mathbf{b}$ for the remaining rare earths ${ }^{1}$ ).

In previous works, bond valence sum (BVS) calculations using $B=0.37$ and $r_{0}=1.686$ for the Ni-O pair yield $2.58(1)$ (Ni1), 3.24(1) (Ni2), and $\delta=0.33(2)$ for $\mathrm{LuNiO}_{3}$ at RT. Using the same set and the structural data of $\mathrm{PrNiO}_{3}$ at $10 \mathrm{~K}$, we obtain 2.80(1), 3.22(2), and 0.21(2) for Ni1, Ni2, and $\delta$, respectively. Qualitatively, these results suggest the existence of a similar long range but somewhat less complete $\mathrm{Ni}^{3+\delta} / \mathrm{Ni}^{3-\delta}$ charge order than that reported for the heavier nickelates. Quantitatively, this should nevertheless be considered with care. On one side, all tabulated BVS parameters have been determined from RT structural data; on the other, the published BVS parameters for the high-valence states of $\mathrm{Ni}$ suffer from large statistical errors. Keeping this in mind, it is perhaps more illustrative to compare the average $\mathrm{Ni}-\mathrm{O}$ distances of the Ni1 and Ni2 polyhedra with those reported for oxides containing single-valent nickel $(+2,+3$, or +4$)$ in octahedral coordination. In Fig. 3 we have represented the dependence of the RT $\left\langle d_{\mathrm{Ni}-\mathrm{O}}\right\rangle$ with the nominal oxidation state in a few families of stoichiometric $\mathrm{Ni}$ oxides. It is to be noted that for compounds with the same nominal Ni valence, $\left\langle d_{\mathrm{Ni}-\mathrm{O}}\right\rangle$ may change by more than $0.05 \AA$ depending on both the type and the magnitude of the distortion displayed by the $\mathrm{NiO}_{6}$ octahedra (see caption). For $\mathrm{PrNiO}_{3}$ and $\mathrm{LuNiO}_{3}$, the average $\mathrm{Ni}-\mathrm{O}$ distance in the metallic state is in both cases larger than in $\mathrm{LaNiO}_{3}$ (lowest pink square) and shorter than in $\mathrm{AgNiO}_{2}$, the two extreme values reported for $\mathrm{Ni}^{3+}$ oxides. In the insulating state, it is important to stress that both $\left\langle d_{\mathrm{Ni1}-\mathrm{O}}\right\rangle$ and $\left\langle d_{\mathrm{Ni2}-\mathrm{O}}\right\rangle$ are well outside of this interval even in the case of $\mathrm{PrNiO}_{3}$ (see Fig. 3), although none of them

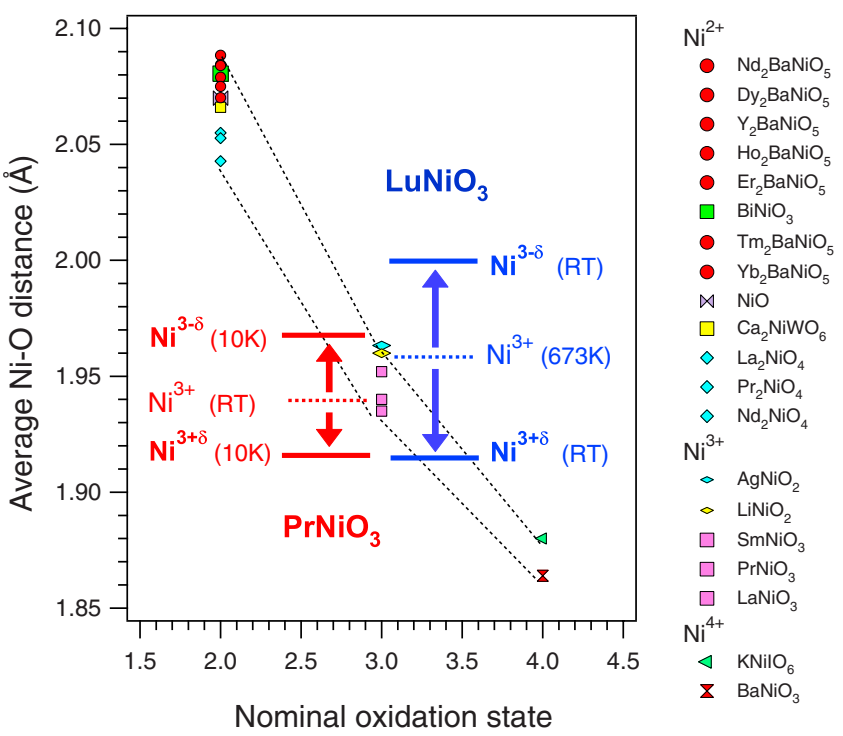

FIG. 3. (Color online) Dependence of the average RT Ni-O distance with the nominal oxidation state. Circles: $\mathrm{Nd}_{2} \mathrm{BaNiO}_{5}$-type (Ref. 20). Rhombuses: $\mathrm{K}_{2} \mathrm{NiF}_{4}$-type (Ref. 21). Squares: perovskitelike compounds (Refs. 22 and 23). Flattened rhombuses: delafossitelike (Ref. 9). $\mathrm{BaNiO}_{3}$ : (Ref. 24). NiO: NaCl-type $\mathrm{KNiIO}_{6}$ :(Ref. $25)$. For a given structural type, the order of the points in the figure coincides with that of the legend.

reached the values reported for pure $\mathrm{Ni}^{2+}$ and $\mathrm{Ni}^{4+}$ oxides.

To investigate whether the incompleteness of the $\mathrm{CD}$ suggested by the values of the average Ni1-O and Ni2-O distances could arise from the simultaneous existence of charge or bond-length fluctuations, either static or dynamic, we have examined the evolution of the Debye-Waller (DW) factors across $T_{\mathrm{MI}}$. Figure 4 shows the thermal evolution of the isotropic $B$ factors of the $\mathrm{Pr}$ and $\mathrm{O}$ sites when refined using the Pbnm space group above and below the transition (black dots). A clear increase is observed in both cases below $T_{\mathrm{MI}}$, which is in agreement with the evolution of the static disorder of the $\mathrm{Ni}$ first coordination shell determined from EXAFS. ${ }^{5}$ The white triangles in the same figure are the values obtained when using the $P 2_{1} / n$ space group below $T_{\mathrm{MI}}$ In contrast to the previous case, a smooth temperature dependence is obtained, indicating that the monoclinic symmetry is able to capture the contribution previously attributed to short_range charge order.

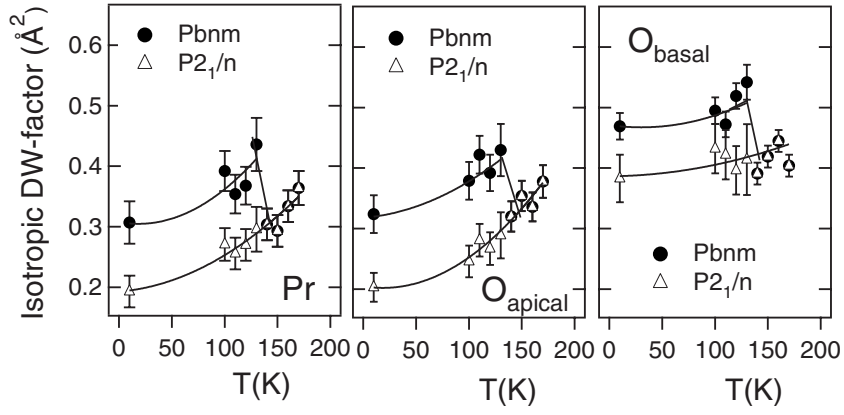

FIG. 4. Temperature dependence of the DW factors $B$ for the Pr and $\mathrm{O}$ sites using space groups $P b n m$ and $P 2_{1} / n$. For $P 2_{1} / n$, $B_{\text {Obasal }}=\left(B_{\mathrm{O} 2}+B_{\mathrm{O} 3}\right) / 2$. 
The existence of dynamic charge fluctuations above and even below $T_{\mathrm{MI}}$ has been suggested from the analysis of EXAFS and IR measurements. In this case, an extra contribution to the DW factors below $T_{\mathrm{MI}}$ and an increase by entering the metallic state will be expected if, as pointed out in Ref. 5, two distinct slow-fluctuating Ni sites coexist in the insulating state with the static charge order and subsist above $T_{\mathrm{MI}}$ as fast charge or bond fluctuations. In Fig. 4 no anomaly at $T_{\mathrm{MI}}$ is evident, at least within our experimental resolution $\left(Q_{\max } \sim 6 \AA\right)$. The isotropic mean-square displacements $\left\langle u^{2}\right\rangle=B / 8 \pi^{2}$ at $10 \mathrm{~K}$ are $0.0025,0.0047$, and $0.0051 \AA^{2}$ for $\mathrm{O} 1, \mathrm{O} 2$, and $\mathrm{O} 3$, respectively. Although absolute DW factor values should be considered with caution, these valuesespecially those of the basal oxygens $\mathrm{O} 2$ and $\mathrm{O} 3$ - are relatively large for zero-point displacements. This result may be compatible with the existence of charge or bond fluctuations which could be at the origin of the reduced $\delta$ value suggested by the values of the average $\mathrm{Ni}-\mathrm{O}$ distances. Further higherresolution $\mathrm{x}$-ray diffraction experiments, preferably on single crystals, would be necessary to confirm it, as well as to provide electron-density maps in order to estimate the degree of delocalization of the Ni-O bonds and the total charge transferred between the two Ni sites.

In conclusion, we have reported high-resolution neutron powder-diffraction data which clearly prove the existence of a static long-range charge order in $\mathrm{PrNiO}_{3}$ below $T_{\mathrm{MI}}$ $=130 \mathrm{~K}$. The magnitude of the associated $\mathrm{CD}$, as measured by the difference between the average $\mathrm{Ni}-\mathrm{O}$ distances in the contracted $\mathrm{Ni}^{3+\delta}$ and expanded $\mathrm{Ni}^{3-\delta}$ sites, appears to be significantly smaller than in $\mathrm{LuNiO}_{3}$. This finding strongly suggests the existence of a mechanism for the electronic localization common to the whole $R \mathrm{NiO}_{3}$ family and a decrease in $\delta$ by approaching the itinerant limit. Analysis of the DW factors shows that the previously reported anomalies at $T_{\mathrm{MI}}$, interpreted as evidence for short-range charge order are absent if the correct space group is used. The relatively large value of the zero-point mean-square displacements for all the atomic sites suggests nevertheless the existence of either a residual dynamic component or a small additional static distortion. In particular, the existence of a noncentrosymmetric space group below $T_{\mathrm{MI}}$ could explain the magnetic structure and would support recent theoretical work predicting the existence of magnetism-induced electric polarization in these compounds. $^{26}$

The beamtime allocation by the ILL is gratefully acknowledged.
${ }^{1}$ P. Lacorre, J. B. Torrance, J. Pannetier, A. I. Nazzal, P. W. Wang, T. C. Huang, and R. L. Siemens, J. Solid State Chem. 91, 225 (1991).

${ }^{2}$ J. A. Alonso, M. J. Martinez-Lope, M. T. Casais, J. L. GarciaMunoz, M. T. Fernandez-Diaz, and M. A. G. Aranda, Phys. Rev. B 64, 094102 (2001).

${ }^{3}$ J. B. Torrance, P. Lacorre, A. I. Nazzal, E. J. Ansaldo, and C. Niedermayer, Phys. Rev. B 45, 8209 (1992).

${ }^{4}$ J. Rodríguez-Carvajal, S. Rosenkranz, M. Medarde, P. Lacorre, M. T. Fernandez-Diaz, F. Fauth, and V. Trounov, Phys. Rev. B 57, 456 (1998).

${ }^{5}$ C. Piamonteze, H. C. N. Tolentino, A. Y. Ramos, N. E. Massa, J. A. Alonso, M. J. Martinez-Lope, and M. T. Casais, Phys. Rev. B 71, 012104 (2005).

${ }^{6}$ M. Acosta-Alejandro, J. M. de Leon, M. Medarde, P. Lacorre, K. Konder, and P. A. Montano, Phys. Rev. B 77, 085107 (2008).

${ }^{7}$ T. Mizokawa, D. I. Khomskii, and G. A. Sawatzky, Phys. Rev. B 63, 024403 (2000).

${ }^{8}$ P. M. Woodward, D. E. Cox, E. Moshopoulou, A. W. Sleight, and S. Morimoto, Phys. Rev. B 62, 844 (2000).

${ }^{9}$ E. Wawrzyńska, R. Coldea, E. M. Wheeler, I. I. Mazin, M. D. Johannes, T. Sorgel, M. Jansen, R. M. Ibberson, and P. G. Radaelli, Phys. Rev. Lett. 99, 157204 (2007).

${ }^{10}$ I. I. Mazin, D. I. Khomskii, R. Lengsdorf, J. A. Alonso, W. G. Marshall, R. M. Ibberson, A. Podlesnyak, M. J. Martinez-Lope, and M. M. Abd-Elmeguid, Phys. Rev. Lett. 98, 176406 (2007).

${ }^{11}$ M. Zaghrioui, A. Bulou, P. Lacorre, and P. Laffez, Phys. Rev. B 64, 081102(R) (2001).

${ }^{12}$ F. P. de la Cruz, C. Piamonteze, N. E. Massa, H. Salva, J. A. Alonso, M. J. Martinez-Lope, and M. T. Casais, Phys. Rev. B 66, 153104 (2002).

${ }^{13}$ M. T. Causa, R. D. Sanchez, M. Tovar, J. A. Alonso, and M. J.
Martinez-Lope, Phys. Rev. B 68, 024429 (2003).

${ }^{14}$ U. Staub, G. I. Meijer, F. Fauth, R. Allenspach, J. G. Bednorz, J. Karpinski, S. M. Kazakov, L. Paolasini, and F. dAcapito, Phys. Rev. Lett. 88, 126402 (2002).

${ }^{15}$ T. Saito, M. Azuma, E. Nishibori, M. Takata, M. Sakata, N. Nakayama, T. Arima, T. Kimura, C. Urano, and M. Takano, Physica B (Amsterdam) 329, 866 (2003).

${ }^{16}$ J. A. Alonso, G. Démazeau, A. Largeteau, D. Kurowski, R.-D. Hoffmann, and R. Pöttgen, Z. Naturforsch., B: Chem. Sci. 61, 346 (2006).

${ }^{17}$ M. Medarde, P. Lacorre, K. Conder, F. Fauth, and A. Furrer, Phys. Rev. Lett. 80, 2397 (1998).

${ }^{18}$ J. S. Zhou, J. B. Goodenough, and B. Dabrowski, Phys. Rev. B 67, 020404(R) (2003).

${ }^{19}$ J. Rodriguez-Carvajal, Physica B (Amsterdam) 192, 55 (1993).

${ }^{20}$ E. García-Matres, J. L. Martínez, and J. Rodríguez-Carajal, J. Solid State Chem. 103, 322 (1993).

${ }^{21}$ M. Fernández-Díaz, Ph.D. thesis, Universidad Autonoma de Madrid, 1991.

${ }^{22}$ J. A. Alonso, M. J. Martinez-Lope, M. T. Casais, J. L. GarciaMunoz, and M. T. Fernandez-Diaz, Phys. Rev. B 61, 1756 (2000).

${ }^{23}$ M. J. Martínez-Lope, J. A. Alonso, M. T. Casais, and M. T. Fernández-Díaz, Z. Naturforsch., B: Chem. Sci. 58b, 127 (2003).

${ }^{24}$ Y. Takeda, F. Kanamaru, M. Shimada, and M. Koizumi, Acta Crystallogr., Sect. B: Struct. Crystallogr. Cryst. Chem. 32, 2464 (1976).

${ }^{25}$ D. B. Currie, W. Levason, R. D. Oldroyd, and M. T. Weller, J. Mater. Chem. 3, 447 (1993).

${ }^{26}$ J. van den Brink and D. I. Khomskii, J. Phys.: Condens. Matter 20, 434217 (2008). 